\title{
Antibiotic Susceptibility Pattern of Mycobacterium tuberculosis
}

\author{
Subba $S,{ }^{1}$ Singh $S,{ }^{1}$ Khagi AR, ${ }^{1}$ Tuladhar R, ${ }^{1}$ Lekhak B, ${ }^{1}$ Khadka DK, ${ }^{2}$ Shrestha KB ${ }^{2}$ \\ ${ }^{1}$ Central Department of Microbiology, Tribhuvan University, Kirtipur, Kathmandu, ${ }^{2}$ National Tuberculosis Center, Bhaktapur, \\ Nepal
}

\section{ABSTRACT}

Background: The emergence and spread of DR and MDR-TB threat global TB control. The susceptibility patterns of M. tuberculosis isolates against anti-tuberculosis drugs informs an important aspect of TB controls and surveillance and analysis of local rates of TB drug resistance helps in the detection and monitoring of the extent of DR and MDR strains, indicating the quality of TB control in the country.

Methods: A cross sectional study was conducted to find out antibiotic susceptibility pattern of Mycobacterium tuberculosis in pulmonary tuberculosis patients at national tuberculosis center. All the samples were stained by auramine fluorochrome method; processed by $\mathrm{NaOH}$ Ogawa Method; primary cultured; subcultured in 2\% Ogawa media; cultured in drug LJ media and finally results observed and interpreted. Here Drug susceptibility test was done on $M$. tuberculosis isolate from each patient by Proportion method as standard protocol.

Results: Ethambutol (66.10\%) was found to be the most effective anti-TB drug according to the susceptibility test followed by RMP (60.33\%), SM (59.66\%) and INH (41.69\%) against M. tuberculosis. Among 45 isolated untreated patients, primary drug resistance was observed in $20 \%$, to two drugs in $17.77 \%$, to three drugs in $11.11 \%$, to four drugs in $6.66 \%$ and primary Multi-drug resistant in $22.22 \%$. Among 250 isolated from previous treated patients, acquired resistance to one drug was found in $23.60 \%$, to two drugs in $12.40 \%$, to three drugs in $16.40 \%$, to four drugs in $18.80 \%$ an acquired Multi-drug resistant in $37.20 \%$. Among 250 treated $68.40 \%(n=171)$ were relapse, $18 \%$ $(\mathrm{n}=45)$ were chronic, $7.6 \%(\mathrm{n}=19)$ were follow-up, 3.2\% $(\mathrm{n}=8)$ were defaulter, and $2.8 \%(\mathrm{n}=7)$ were treatment failure. MDR-TB was found the highest in chronic cases $(64.44 \%)$ followed by follow-up case (47.36\%), treatment failure cases $(42.85 \%)$, relapse cases $(27.48 \%)$ and default cases $(12.5 \%)$. A statistical analysis reveals significant relationship between prior history of treatment and the development of drug resistance. However, no significant relationship between age and sex with the emergence of drug resistance isolates.

Conclusion: MDR-TB was found the highest in chronic cases $(64.44 \%)$ followed by follow-up case (47.36\%), treatment failure cases $(42.85 \%)$, relapse cases $(27.48 \%)$ and Default cases $(12.5 \%)$.

Key words: multi drug, relapse, resistant, sensitivity, tuberculosis

Correspondence: S Subba, Central Department of Microbiology, Tribhuvan University, Kirtipur, Kathmandu, Nepal. 
Antibiotic Susceptibility Pattern of Mycobacterium tuberculosis

\section{INTRODUCTION}

Tuberculosis (TB) is a treatable and preventable disease. ${ }^{1}$ It is an infectious bacterial disease caused by Mycobacterium an acid fast bacilli. ${ }^{2}$ The SAARC region accounts more than $29 \%$ of global burden of tuberculosis with 0.6 million deaths every year and 2 million new cases annually. ${ }^{3}$ Tuberculosis is a socio-medical problem. ${ }^{4}$ Ziehl-Neelson Staining or by Fluoro-chrome staining allows highly accurate diagnosis widely available, simple and multi-purpose equipment. ${ }^{5}$ Culture increases the number of tuberculosis cases found, often by $30-50 \%$ and detects cases earlier, often before they become infectious. ${ }^{6}$ Most of the recent advances in the laboratory diagnosis of TB BY rapid culture, identification, and susceptibility systems. ${ }^{7}$

Drug resistant tuberculosis is a case of tuberculosis (usually pulmonary) excreting bacilli resistant to one or more anti-tuberculosis drugs. MDR-TB is resistant to at least Isoniazide and Rifampicin, the main antituberculosis drugs. ${ }^{8}$

DOTS strategy as one of the most cost effective health interaction and recommends that effective TB treatment be a part of the essential clinical service package available in primary health care. In Nepal, DOTS strategy has been implemented since 1996 and has already reduced the number of deaths. ${ }^{9}$

\section{METHODS}

A cross sectional study conducted to find out antibiotic susceptibility pattern of Mycobacterium tuberculosis in pulmonary tuberculosis patients was at National Tuberculosis Centre from September 2005 to May 2006. A total of 295 clinically suspected patients having sputum positive diagnosed by fluorescent microscopy were included in the study. The microscopy produced bright yellowish bacteria against a dark background. The sputum samples were decontaminated and cultured on $2 \%$ modified Ogawa media and smear examination was done from pellet. The $M$. tuberculosis bacteria were confirmed by different biochemical tests. The culture positive isolates were tested for anti-TB drug susceptibility testing by Proportional Method as standard protocol.

Each strain was tested against four antibiotics at the following concentrations: Isoniazid (INH) at $0.25 \mu \mathrm{m} / \mathrm{ml}$ and $8 \mu \mathrm{m} / \mathrm{ml}$; Ethambutol (EMB) at $1 \mu \mathrm{m} / \mathrm{ml}$ and $2 \mu \mathrm{m} / \mathrm{ml}$; Streptomycin(SM) at $4 \mu \mathrm{m} / \mathrm{ml}$ and $8 \mu \mathrm{m} / \mathrm{ml}$ and Rifampicin (RMP) at $20 \mu \mathrm{m} / \mathrm{ml}$ and $40 \mu \mathrm{m} / \mathrm{ml}$.

The LJ medium is used for all the resistance tests. Working drug dilutions was prepared on the day of use; $1 \mathrm{ml}$ of working solution added to $500 \mathrm{ml}$ of LJ medium will yield final drug concentrations equivalent to the different critical concentrations. The medium is distributed in volumes of $6-8 \mathrm{ml}$ in sterile $17 \mathrm{~mm} \times 170$ $\mathrm{mm}$ screw-capped test tubes, coagulated at $85{ }^{\circ} \mathrm{C}$ for 45 minutes, and allowed to cool at room temperature for 24 hours; the screw caps are then tightened and the tubes stored at $4{ }^{\circ} \mathrm{C}$. The control medium without drugs is prepared at the same time as the drug-containing media. The period of validity of the media stored at 4 ${ }^{\circ} \mathrm{C}$ is 2 months.

\section{RESULTS}

A total of 295 suspected PTB cases attending at NTC was included in the study. The sputum samples from patients under study were subjected to test for Fluorochrome staining, culture and antibiotic susceptibility testing for culture positive isolates.

Among the studied 295 cases, $73.89 \%$ ( $n=218$ ) were male and $26.10 \%(n=77)$ were female in the age group from 11 year to 88 year. The study showed that the highest number was seen in the age group 21-30, followed by 31-40 (Table 1).

Among 295 M. tuberculosis isolates, 41.69\% ( $n=123)$ were sensitive to INH; $59.66 \%(n=176)$ were sensitive to SM; $60.33 \%(n=178)$ were sensitive to RMP; and $66.10 \%$ $(n=195)$ were sensitive to EMB (Table 2).

Similarly, $58.30 \% \quad(n=172)$ were resistant to $\mathrm{INH}: 40.33 \%$ $(n=119)$ were resistant to $S M ; 9.66 \% \quad(n=117)$ were resistant to RMP; and $33.89 \%(n=100)$ were resistant to EMB.

Among 295 cultures positive M. tuberculosis isolates studied from drug susceptibility testing, resistance to one or more anti-drugs were exhibited.

Out of 295 isolates, 45 isolates were from untreated patients and 250 isolates were from treated patients.

Among the untreated patients, $35.55 \%(n=16)$ were sensitive to all the 4 drugs whereas the remaining were resistant to two or more drugs. Resistant to only one drug was shown by $20 \%(n=9)$ of the isolates. Resistant to two drugs was shown by $17.77 \%(n=8)$, three drugs to $11.11 \%(n=5)$ and four drugs to $6.66 \%(n=3)$ of the isolates (Table 3).

Out of the 250 treated TB patients, 25.20\% ( $n=63)$ were sensitive to all the four anti-TB drugs and the remaining187 isolates were resistant to one or more drugs. Resistant to one drug was found in $23.6 \%(n=59)$; two drugs to $12.4 \%(n=31)$ and three drugs to $16.4 \%$ $(n=41)$. 
Multi Drug Resistance (MDR) in untreated TB patients were found in $22.22 \%(n=10)$ and in treated TB patients was $37.20 \%(\mathrm{n}=93)$.

Among 250 treated cases, $68.4 \%(n=171)$ were relapses; $18 \%(n=45)$ were chronic; $7.6 \%(n=19)$ were followup; $3.2 \%(n=8)$ were defaulters; and $2.8 \%(n=7)$ were Treatment failure PTB cases (Table 4).

The highest percentage of MDR TB was from chronic (64.44\%) followed by follow-up (47.36\%), Treatment failure $(42.85 \%)$, relapse $(27.48 \%)$ and defaulter $(12.5 \%)$.

The primary drug resistance (PDR) to one drug was 20\%, to two drugs was $17.77 \%$, to 3 drugs was $11.11 \%$ to four drugs was $6.66 \%$ and primary MDR was in $22.22 \%$ of the isolates (Table 5). Similarly, acquired drug resistance to one drug was $23.60 \%$,to two drugs was $12.40 \%$, to three rugs was $16.40 \%$, to four drugs was $18.80 \%$ and acquired MDR was in $37.20 \%$ of the isolates.

The highest number of Mycobacterium spp was found to be maximum in age group above 71 i.e. $(100 \%, n=7)$ followed by $80 \%(n=36)$ in $31-40$ age group, $78.94 \%(n=15)$ in age group $11-20,74.19 \%(n=46)$ in $21-30$ age group, 69.23\% ( $n=27)$ in $41-50$ age group, $61.90 \%(n=13)$ in $51-61$ age group and $8.33 \%(n=2)$ in age group $61-70$ in case of male (Table 6).

In case of female patients, the highest number of resistant was found in age group 41-50 76.92\% ( $n=10)$ followed by $73.91 \%(n=17)$ in $21-30$ age group, $66.66 \%$ $(n=12)$ in age group $11-20,31-40$ and $61-70,40 \%(n=2)$ in 51-60 age group (Table 7). The study demonstrated that age and sex were not significantly related to drug resistance $(\mathrm{P}<0.05)$.

The study demonstrated that age and sex were not significantly related to drug resistance. Among 79 drug sensitive strains isolated from PTB patients, $40.50 \%$ $(n=32)$ were isolated from those patients who had previous cases of TB in his/her family and $59.50 \%(n=47)$ had no previous cases of PTB in his/her family.

\section{DISCUSSION}

Tuberculosis has become a grave concern in all part of the world because of recent resurgence of TB. Reasons of this resurgence have been identified by WHO as mainly due to HIV pandemic, less health priority given to the disease and significant increase of multidrug resistant tubercle bacilli as a result of inadequate treatment. The causative agent was discovered more than 100 years ago and highly effective drugs and vaccines are available making TB a preventable and curable disease. It remains as the most significant cause of morbidity and mortality due to a single infectious agent in the world.

Tuberculosis is one of the major public health problems in the third world countries with approximately estimated $60 \%$ of the adult population being infected with TB. In Nepal, about $45 \%$ of the total population is infected with TB. Every year 40,000 people develop active TB of whom 20,000 have infectious pulmonary disease. Nepal, by estimated number of cases, is ranked at 27 globally. Despite the expansion and implementation of a much improved National Tuberculosis Programme (NTP) through DOTS Strategy throughout the country,60007000 people still die from TB each year in Nepal. ${ }^{9}$

The main objective of this study is to know the resistance pattern of the anti-TB drugs in PTB patients. A total 295 cases were included in this study carried out from September 2005 to May 2006. Out of 295 cases, 250 cases were previously treated cases and 45 were untreated cases. During this study, among the 295 cases of TB, $73.89 \%(n=218)$ males were found higher in number than female $26.10 \%(n=77)$ in age group discussion from 11 to above 80 year. The highest number of cases belonged to the age group 21-30 (29.81\%). This finding was concordant with similar studies in other countries. In a similar study in Italy, Ponticiella et al. (1997) reported $82.2 \%$ males and $17.8 \%$ of females among 90 active PTB cases; Blumberg et al (1991-1997) in Atlanta, USA reported $74 \%$ of the male and $26 \%$ of the female TB cases among 1536 cases. Likewise, in Archangeh, Russia, Toungoussova et al in Korea reported $66.49 \%$ if the male and $34.31 \%$ of the female TB cases among 2486 cases. Kuban et al in Cameroon, Yaunde, reported 65.76\% of male and $34.25 \%$ of the female TB cases among 111 cases; Riantawan et al in Thailand reported $77 \%$ male and $23 \%$ of the female cases among 1441 cases..$^{10-15}$ Tuberculosis Control Programme, Nepal reported $66.77 \%$ male and $33.23 \%$ female of TB cases among 14,384 newly diagnosed TB cases during 2002/2003. All above findings are consistent with this study. Shrestha et al reported $47 \%$ males and $3.05 \%$ of female TB cases in histopathological specimens at Tribhuvan University Teaching Hospital. ${ }^{12}$ Smith reported that as in most countries of the world, in Nepal, the reported incidence of TB is higher in man than women. Rijal reported that the study conducted at NTC, among the 325 cases, $75.69 \%$ were male and $24.30 \%$ were female. ${ }^{12-19}$ Thus the incidence of TB was found higher in male than female patients; male patients are affected more than female patients. According to the significance test, the prevalence of TB in male and female was found to be statistically significant. These possible factors explain the gender differences observed, the most commonly accepted being that women are less exposed to infection than men. The second might be the biological difference, such as an increased susceptibility 
Antibiotic Susceptibility Pattern of Mycobacterium tuberculosis

in male. Finally, infected women may progress more frequently to disease and die more rapidly, leaving a cohort with a low prevalence of infection.

In this study, out of 295 culture positive isolates, 15.25\% $(n=45)$ isolates from the untreated TB cases and $84.74 \%$ $(n=250)$ isolates from the treated TB cases. The study showed that $26.77 \%(n=79)$ were sensitive to all the four drugs and $68.81 \%(n=203)$ cases were drug resistant to one or more drugs. Monoresistance to INH, SM, RMP and EMB was detected in 41(13.89\%), 19(6.44\%), 1(0.33\%) and $7(2.37 \%)$ strains respectively; and $23.05 \%$ to a total single drug resistance. Similarly, resistance to two drugs was detected in 39 strains(13.22\%); resistant to three drugs was detected in 46 strains $(16.94 \%)$. The level of Multi-drug resistant cases were found to be $34.91 \%$ (22.22\% Primary MDR and 37.20\% Acquired MDR).

The highest rates of drug resistance were discovered for isoniazid and streptomycin. In both new and treated cases, $13.33 \%$ and $14 \%$ of the strains isolated were resistant to isoniazid respectively; whereas $6.66 \%$ and $6.40 \%$ of the strains isolated from new and previously treated patients were resistant to streptomycin respectively.

The initial drug resistance case was found in $8.47 \%$ to one or more of the four anti-TB drugs was found to be $60.33 \%$. In addition, the primary drug resistance(PDR) to one drug was $20 \%$, to two drugs was $17.77 \%$, to 3 drugs was $11.11 \%$ and to four drugs was $6.66 \%$. And the acquired drug resistant to one drug was $23.60 \%$, to two drugs was $12.40 \%$, to three drugs was $16.40 \%$ and to four drugs was $18.80 \%$. The primary MDR was found to $22.22 \%$ of the isolates and the acquired MDR was in $37.20 \%$ of isolates. This finding of this study is similar with the latest third surveillance report of WHO 2004 in Nepal. The report revealed that $1.3 \%$ and $20.5 \%$ of the new and old cases had MDR in Nepal. The rate of acquired MDRTB was higher $(19.25 \%)$ than the rate of primary MDR-TB (2.63\%).

Out of 295 culture positives isolates obtained from the PTB patients, 171 isolates were from relapse cases, 45 isolates from chronic cases, 19 isolates from followup cases, 8 isolates from default cases and 7 isolates from treatment failure cases. The highest percentage of MDR was obtained from the chronic cases (64.44\%) followed by follow-up cases (47.36\%), treatment failure cases $(42.85 \%)$, relapse cases $(27.48 \%)$ and default cases (12.5\%).

The finding of this study is in agreement with other studies conducted at different places. Al Marri in the state of Qatar reported that $85 \%$ of the cases were sensitive to anti-TB drugs and $15 \%$ cases were resistant to one or more
anti-TB drugs among 406 cases of PTB. ${ }^{14}$ In West Province of Cameroon, the level of initial drug resistance was found in $14.28 \%$ and acquired drug resistance was found in $0.74 \%$, the rate of MDR was found $0.98 \%$ (0.49\%initial MDR and $1 \%$ for acquired MDR); Kuban et al. reported 4.1\%MDR case among 566 isolates, 15.2\% initial drug resistance and $11.6 \%$ acquired drug resistance and $1.06 \%$ and acquired MDR cases 3\%). ${ }^{15}$ Indian studies showed that $3.4 \%$ of the new cases and $25 \%$ of the old cases had MDR-TB (WHO,2000). ${ }^{23}$ The anti-TB drug sensitivity test conducted in Nepal 1987-1990 revealed that $1.6 \%$ and $9.6 \%$ of the new and old cases respectively had MDRTB.GC et al.(2001) found $8.57 \%$ of initial MDR cases and $100 \%$ of acquired MDR cases; Bhattarai et al. (2003) obtained $4.16 \%$ of primary MDR cases and $5 \%$ of acquired MDR cases. Similarly, Rijal et al. (2003) found primary MDR in $2.63 \%$ of the isolates and acquired MDR in $19.25 \%$ of isolates. ${ }^{17-23}$ The alarming increment in MDR-TB cases may be owing to late identification of suspected MDRTB cases. Identification of all cases of MDR-TB would require culture and susceptibility testing of tuberculosis suspects, an ideal that is unachievable in developing countries like Nepal. The culture and sensitivity facilities for M. tuberculosis in our country are country are only in National Tuberculosis Centre (NTC) and GermanyNepal Tuberculosis. Project (GENETUP). Other reasons for increase in MDR-TB may have smear negative TB and hence may even remain undiagnosed with tuberculosis. Even among those with smear positive disease, initial response to treatment may be good, and MDR-TB may not be suspected in some cases.

Our study revealed that there were high rates of initial drug resistance against isoniazid and streptomycin among both new and treated cases. This may be due to low cost and widespread use in the treatment of TB. The most significant finding of our study was the low frequency of primary resistance to rifampicin which is a good indicator for success of DOTS. Mono resistance to Rifampicin was not observed at all in new cases. Resistance to Rifampicin predicted resistance to isoniazid and streptomycin and served as marker of MDR.

The high rates of resistance among new cases indicated that drug resistant strains are circulating and are being transmitted from patient to patient in our country Nepal. Transmission of already resistant strains as a serious problem and threat, as it is different to treat patients infected with drug resistance, it is important for a TB control programme to have reliable laboratory facilities for susceptibility testing of $\mathrm{M}$.tuberculosis isolates.

This study showed there were no any relation of age and sex, with drug resistance. The result of the present study was in agreement with the study of Al-Marri in Qatar; Warndroff et al in Karongo District, Malavi also supports 
our study that neither nor acquired drug resistance were associated with sex or age. ${ }^{14,18}$

Likewise, the family history of TB cases, smoking and alcoholic habit of the patients did not show any significant relation with drug resistance. This study was similar to the study of Leung in Hong Kong; and Toungoussova et al., 2002; Archangels in Russia. ${ }^{10,20}$

Increase cases of MDR-TB are a global problem. MDR-TB can be cured by the effective implementation of DOTS strategy. Regular monitoring of MDR-TB and policy in accordance with the operational research finding enables the controls and drug quality assessment is helpful for emerging MDR-TB.

In 1997 the World Health Organization (WHO), the International Union Against Tuberculosis and Lung Disease (IUATLD) and partners world-wide released the first report of the global project on anti-tuberculosis drug resistance. ${ }^{22}$ The data generated in this report were reinforced in a recently published second report. Directly observed treatment short-course (DOTS), the WHO strategy for TB control cures virtually all patients with drug-susceptible TB and some drug resistant TB through the administration of short-course chemotherapy with first-line drugs.

However, patients with multidrug-resistant (MDR) tuberculosis (TB) to at least isoniazid and rifampicin are more likely to fail short-course chemotherapy. In recent years there has been encouraging evidence that patients with MDR TB can be cured with appropriate management based on second-line drugs. Unfortunately, second-line drugs are inherently more toxic and less effective than first-line drugs and reliable assessment of drug resistance is an essential prerequisite for appropriate use. Treatment is prolonged and significantly more expensive. Accurate laboratory drug susceptibility testing (DST) data to second-line drugs will support clinical decision making and help to prevent the emergence of further drug resistance in patients with MDR TB. In order to meet the challenges posed by MDR TB, the WHO established the DOTS-Plus initiative to assess the feasibility and cost-effectiveness of using second-line drugs to manage patients with MDR TB primarily in middle and low-income countries.

DOTS-Plus is needed in areas where MDR-TB has emerged due to previous inadequate TB control programmes. Therefore, DOTS-Plus pilot projects are only recommended in settings where the standard DOTS strategy is fully in place to protect against the creation of further drug resistance. DOTS-Plus is designed to cure MDR-TB using second-line TB drugs. These drugs should be stored and dispensed at specialized health centers with appropriate facilities and well-trained staff. It is vital that DOTS-Plus pilot projects follow WHO recommendations in order to minimize the risk of creating drug resistance to second line TB drugs. DOTSPlus works as a supplement to standard DOTS-based TB programmes already in place.

If patients failing DOTS are presumed to have MDR-TB, and if drug-susceptibility testing is limited, they might be placed on an empirical treatment regimen consisting of second-line TB drugs. Under DOTS Plus, they must endure an additional two years of daily, observed combination therapy, including injectable antibiotics, which can produce unpleasant side-effects. As of July 2002, the Green Light Committee (GLC) had approved seven pilot projects to implement the DOTS-Plus strategy, and is currently reviewing five further applications. Preliminary results from those programmes already under way show percentages of culture negativization to be between 46 and 79 percent. Continued support for these projects - together with the implementation of new programmes in other countries - will contribute to the building of a sound policy for the control of MDR-TB.

Estonia's country-wide DOTS-Plus programme began in March 2001, and allows for the enrolment of 200 patients over a two-year period. Preliminary results show a sputum negativization of 46 percent of patients after six months of treatment. The pilot project has become a leverage tool to promote the expansion of the DOTS strategy in Estonia.

Latvia began to implement DOTS fully in 1997. However, poor case management in the past and the overcrowded conditions of TB wards still helped to make Latvia the country with the second highest MDR-TB rate in the world. The proportion of MDR-TB among new TB patients in this country is 9.5 percent. On February 2001, the GLC approved a countrywide DOTS-Plus pilot project.

Strengthening MDR-TB control now through DOTS-Plus will help to reduce morbidity, mortality and transmission due to MDR-TB. By directing MDR-TB patients to effective treatment protocols now, we are saving direct costs. And by controlling the primary cycle of MDR-TB transmission now, we are saving future funds and indirect costs that would otherwise have to be diverted into treatment for both sick individuals and those that they infect.

\section{CONCLUSION}

MDR-TB was found the highest in chronic cases $(64.44 \%)$ followed by follow-up case $(47.36 \%)$, treatment failure cases $(42.85 \%)$, relapse cases $(27.48 \%)$ and Default cases (12.5\%). A statistical analysis reveals no significant relationship between age and sex with the emergence of drug resistant isolates. 
Antibiotic Susceptibility Pattern of Mycobacterium tuberculosis

\begin{tabular}{|c|c|c|c|c|c|c|c|}
\hline \multirow{2}{*}{ S.N. } & \multirow{2}{*}{ Age group } & \multicolumn{2}{|c|}{ Male } & \multicolumn{2}{|c|}{ Female } & \multicolumn{2}{|c|}{ Total } \\
\hline & & No. & $\%$ & No. & $\%$ & No. & $\%$ \\
\hline 1 & $11-20$ & 19 & 6.44 & 18 & 6.10 & 37 & 12.54 \\
\hline 2 & $21-30$ & 62 & 21.02 & 23 & 7.79 & 85 & 28.81 \\
\hline 3 & $31-40$ & 45 & 15.25 & 16 & 5.42 & 61 & 20.68 \\
\hline 4 & $41-50$ & 39 & 13.22 & 13 & 4.41 & 52 & 17.63 \\
\hline 5 & $51-60$ & 21 & 7.12 & 5 & 1.69 & 26 & 8.81 \\
\hline 6 & $61-70$ & 24 & 8.14 & 3 & 1.02 & 27 & 9.15 \\
\hline 7 & $71-80$ & 6 & 2.03 & 0 & 0.00 & 6 & 2.03 \\
\hline 8 & Above 80 & 1 & 0.34 & 0 & 0.00 & 1 & 0.34 \\
\hline
\end{tabular}

\begin{tabular}{|c|c|c|c|c|c|c|}
\hline \multirow{2}{*}{ S.N. } & \multirow{2}{*}{ Antibiotics } & \multirow{2}{*}{ No. of isolates } & \multicolumn{2}{|c|}{ Sensitive } & \multicolumn{2}{|c|}{ Resistant } \\
\hline & & & No. & $\%$ & No. & $\%$ \\
\hline 1 & $\mathrm{INH}$ & 295 & 123 & 41.69 & 172 & 58.30 \\
\hline 2 & SM & 295 & 176 & 59.66 & 119 & 40.33 \\
\hline 3 & RMP & 295 & 178 & 60.33 & 117 & 39.66 \\
\hline 4 & EMB & 295 & 195 & 66.10 & 100 & 33.89 \\
\hline
\end{tabular}

\begin{tabular}{|c|c|c|c|c|c|c|c|c|}
\hline \multirow{2}{*}{ SN } & \multirow{2}{*}{$\begin{array}{l}\text { Drug susceptibility testing } \\
\text { result }\end{array}$} & \multicolumn{2}{|c|}{ Untreated TB patients } & \multicolumn{3}{|c|}{ Treated TB patients } & \multirow{2}{*}{ Total } & \multirow{2}{*}{$\begin{array}{l}\text { Total in } \\
\text { Percent }\end{array}$} \\
\hline & & No. & $\%$ & No. & $\%$ & & & \\
\hline 1 & Total tested & 45 & 15.25 & 250 & 84.74 & & 295 & \\
\hline 2 & Sensitive to all 4 Drugs & 16 & 35.55 & 63 & 25.20 & & 79 & $26.77 \%$ \\
\hline \multirow[t]{5}{*}{3} & Resistant to 1 Drug & & & & & & & \\
\hline & INH & 6 & 13.33 & 35 & 14.00 & & 41 & $13.89 \%$ \\
\hline & SM & 3 & 6.66 & 16 & 6.40 & & 19 & $6.44 \%$ \\
\hline & RMP & 0 & 0 & 1 & 0.40 & & 1 & $0.33 \%$ \\
\hline & EMB & 0 & 0 & 7 & 2.80 & & 7 & $2.37 \%$ \\
\hline \multirow[t]{6}{*}{4} & Resistant to 2 Drugs & & & & & & & \\
\hline & INH+RMP & 2 & 4.44 & 9 & 3.60 & & 11 & $3.72 \%$ \\
\hline & $\mathrm{INH}+\mathrm{SM}$ & 1 & 2.22 & 5 & 2.00 & & 6 & $2.03 \%$ \\
\hline & $\mathrm{RMP}+\mathrm{SM}$ & 3 & 6.66 & 3 & 1.20 & & 6 & $2.03 \%$ \\
\hline & $\mathrm{INH}+\mathrm{EMB}$ & 2 & 4.44 & 14 & 5.60 & & 16 & $5.42 \%$ \\
\hline & $\mathrm{SM}+\mathrm{EMB}$ & 0 & 0 & 0 & 0 & & 0 & $0 \%$ \\
\hline \multirow[t]{5}{*}{5} & Resistant to 3 Drugs & & & & & & & \\
\hline & $\mathrm{INH}+\mathrm{RMP}+\mathrm{SM}$ & 3 & 6.66 & 24 & 9.60 & 27 & & $9.15 \%$ \\
\hline & $\mathrm{RMP}+\mathrm{SM}+\mathrm{EMB}$ & 0 & 0 & 0 & 0 & 0 & & $0 \%$ \\
\hline & $\mathrm{RMP}+\mathrm{INH}+\mathrm{EMB}$ & 2 & 4.44 & 11 & 4.40 & 13 & & $4.40 \%$ \\
\hline & $\mathrm{INH}+\mathrm{SM}+\mathrm{EMB}$ & 0 & 0 & 6 & 2.40 & 6 & & $2.03 \%$ \\
\hline 6 & Resistant to all Drugs & 3 & 6.66 & 47 & 18.80 & 50 & & $16.94 \%$ \\
\hline 7 & $\begin{array}{l}\text { Multi-Drug Resistance (MDR) at } \\
\text { least INH + RMP }\end{array}$ & 10 & 22.22 & 93 & 37.20 & 103 & & $34.91 \%$ \\
\hline
\end{tabular}


Antibiotic Susceptibility Pattern of Mycobacterium tuberculosis

\begin{tabular}{|c|c|c|c|c|c|c|c|c|c|c|c|}
\hline \multirow{2}{*}{ S.N. } & \multirow{2}{*}{$\begin{array}{l}\text { Drug susceptibility } \\
\text { testing result }\end{array}$} & \multicolumn{2}{|c|}{ Relapse } & \multicolumn{2}{|c|}{ Chronic } & \multicolumn{2}{|c|}{ Follow-Up } & \multicolumn{2}{|c|}{ Default } & \multicolumn{2}{|c|}{ Treatment failure } \\
\hline & & No. & $\%$ & No. & $\%$ & No. & $\%$ & No. & $\%$ & No. & $\%$ \\
\hline 1 & Total tested & 171 & 68.4 & 45 & 18 & 19 & 7.6 & 8 & 3.2 & 7 & 2.8 \\
\hline 2 & Sensitive to all 4 Drugs & 45 & 26.31 & 5 & 11.11 & 6 & 31.57 & 4 & 5 & 2 & 28.57 \\
\hline \multirow{5}{*}{3} & \multicolumn{11}{|l|}{ Resistant to 1 Drug } \\
\hline & INH & 28 & 16.37 & 3 & 6.66 & 2 & 10.52 & 1 & 12.5 & 1 & 14.28 \\
\hline & SM & 13 & 7.60 & 2 & 4.44 & 0 & 0 & 1 & 12.5 & 0 & 0 \\
\hline & RMP & 1 & 0.58 & 0 & 0 & 0 & 0 & 0 & 0 & 0 & 0 \\
\hline & EMB & 9 & 5.26 & 1 & 2.22 & 0 & 0 & 0 & 0 & 0 & 0 \\
\hline \multirow[t]{6}{*}{4} & \multicolumn{11}{|l|}{ Resistant to 2 Drugs } \\
\hline & $\mathrm{INH}+\mathrm{RMP}$ & 5 & 2.92 & 0 & 0 & 1 & 5.26 & 0 & 0 & 1 & 14.28 \\
\hline & $\mathrm{INH}+\mathrm{SM}$ & 3 & 1.75 & 0 & 0 & 0 & 0 & 1 & 12.5 & 0 & 0 \\
\hline & $\mathrm{RMP}+\mathrm{SM}$ & 3 & 1.75 & 1 & 2.22 & 0 & 0 & 0 & 0 & 0 & 0 \\
\hline & $\mathrm{INH}+\mathrm{EMB}$ & 12 & 7.01 & 1 & 2.22 & 0 & 0 & 0 & 0 & 0 & 0 \\
\hline & $\mathrm{SM}+\mathrm{EMB}$ & 0 & 0 & 0 & 0 & 0 & 0 & 0 & 0 & 0 & 0 \\
\hline \multirow[t]{5}{*}{5} & \multicolumn{11}{|l|}{ Resistant to 3 Drugs } \\
\hline & $\mathrm{INH}+\mathrm{RMP}+\mathrm{SM}$ & 12 & 7.01 & 10 & 22.22 & 1 & 5.26 & 0 & 0 & 1 & 14.28 \\
\hline & $\mathrm{RMP}+\mathrm{SM}+\mathrm{EMB}$ & 1 & 0.58 & 0 & 0 & 0 & 0 & 0 & 0 & 0 & 0 \\
\hline & $\mathrm{RMP}+\mathrm{INH}+\mathrm{EMB}$ & 5 & 2.92 & 6 & 13.33 & 0 & 0 & 0 & 0 & 0 & 0 \\
\hline & $\mathrm{INH}+\mathrm{SM}+\mathrm{EMB}$ & 2 & 1.16 & 2 & 4.44 & 1 & 5.26 & 0 & 0 & 0 & 0 \\
\hline 6 & Resistant to all Drugs & 25 & 14.61 & 13 & 28.88 & 7 & 36.84 & 1 & 12.5 & 1 & 14.28 \\
\hline 7 & $\begin{array}{l}\text { Multi-Drug Resistance } \\
\text { (MDR) at least INH+RMP }\end{array}$ & 47 & 27.48 & 29 & 64.44 & 9 & 47.36 & 1 & 12.5 & 3 & 42.85 \\
\hline
\end{tabular}

The highest percentage of MDR TB was from chronic $(64.44 \%)$ followed by follow-up (47.36\%), treatment failure (42.85\%), relapse $(27.48 \%)$ and defaulter $(12.5 \%)$.

Table 5. Comparison of primary and acquired anti-TB drug resistance.

\begin{tabular}{|lccccc|}
\hline Drug resistance & 1 Drug & 2 Drugs & 3 Drugs & 4 Drugs & MDR \\
\hline Primary drug resistance $(n=45)$ & 20.00 & 17.77 & 11.11 & 6.66 & 22.22 \\
Acquired drug resistance $(n=250)$ & 23.60 & 12.40 & 16.40 & 18.80 & 37.20 \\
\hline
\end{tabular}

\section{ACKNOWLEDGEMENT}

I express my cordial gratitude to Dr. Anjana Singh, and other staffs Central Department of Microbiologyfor their kind help. I am especially thankful to Mr. Ram Babu Shrestha, Bhola Chaudhary and other staffs of National
Tuberculosis Center and my special thanks to Mr. Hari Maharjan, Librarian of SAARC Tuberculosis Centre. I would like to express special thanks to Mr. Rajendra Chaudhary, Mrs. Hema Subba and Mr. Bhimhang Limbu for their co-operation, help and suggestion in completing my work. 
Antibiotic Susceptibility Pattern of Mycobacterium tuberculosis

\begin{tabular}{|c|c|c|c|c|c|c|}
\hline \multirow{2}{*}{ S.N. } & \multirow{2}{*}{ Age group } & \multirow{2}{*}{ No. of isolates } & \multicolumn{2}{|c|}{ Sensitive } & \multicolumn{2}{|c|}{ Resistant } \\
\hline & & & No. & $\%$ & No. & $\%$ \\
\hline 1 & $11-20$ & 19 & 4 & 21.05 & 15 & 78.94 \\
\hline 2 & $21-30$ & 62 & 16 & 25.80 & 46 & 74.19 \\
\hline 3 & $31-40$ & 45 & 9 & 20.00 & 36 & 80.00 \\
\hline 4 & $41-50$ & 39 & 12 & 30.76 & 27 & 69.23 \\
\hline 5 & $51-60$ & 21 & 8 & 38.09 & 13 & 61.90 \\
\hline 6 & $61-70$ & 24 & 22 & 91.66 & 2 & 8.33 \\
\hline 7 & Above 71 & 7 & 0 & 0.00 & 7 & 100 \\
\hline Total & & 217 & 71 & & 146 & \\
\hline
\end{tabular}

Table 7. Age wise distribution of resistant M. tuberculosis in female

\begin{tabular}{|lcccccc|}
\hline \multirow{2}{*}{ S.N. } & Age group & No. of isolates & No. & $\%$ & No. & $\%$ \\
\hline 1 & $11-20$ & 18 & 6 & 33.33 & 12 & 66.66 \\
2 & $21-30$ & 23 & 6 & 26.08 & 17 & 73.91 \\
3 & $31-40$ & 16 & 4 & 25.00 & 12 & 66.66 \\
4 & $41-50$ & 13 & 3 & 23.07 & 10 & 76.92 \\
5 & $51-60$ & 5 & 3 & 60.00 & 2 & 40.00 \\
6 & $61-70$ & 3 & 1 & 33.33 & 2 & 66.66 \\
\hline Total & & 78 & 23 & & 55 & \\
\hline \hline
\end{tabular}

\section{REFERENCES}

1. Banavaliker JN, Bhalotra B, Sharma DC, Goel MK, Khandekas PS, Bose M Identification of M. tuberculosis by PCR in clinical specimens. Indian J tuberc 1998;45-15.

2. Park K. Park's Textbook of Preventive and Social Medicine. India: M/S Banarsidas Bhanol Publisher; 2000. p. 137-150.

3. Bam DS, Rahman M, Samaratunga M. Involving Medical colleges in Tuberculosis and HIV control. STC Newsletter 2002 JanJune;13(1):9-10.

4. STC News Letter (2001) Jan-Mar.

5. Deun AV. Role of the Microscopy Network in the NTP. STC Newsletter 2001 Jan-March;1:18-23

6. World Health Organization. Laboratory services in Tuberculosis control Part III: microscopy. Geneva: World Health Organization; 1998.

7. World Health Organization. Bull World Health Organ. Int J Public Health 2002;80(6):426-523.

8. Rijal B, Rahman M and Bam DS (2002) Multi-Drug Resistant Tuberculosis: an overview of the SAARC Region. STC Newsletter 2002 Jan-June;12(1):13-14.

9. National Tuberculosis Control Programme Annual Report, Thimi Bhaktapur: National Tuberculosis centre; 2001-2002.
10. Toungousssova OS, Caugant DA, Sandven PB, June G. Drug resistance of Mycobacterium tuberculosis strains isolated from patients with pulmonary tuberculosis in Archangels, Russia. The international journal of TB and lung disease 2002;6:5406-14.

11. Raintawan P, Punnotok J, Chaisuksuwan R, Prasugarit V (1997) Resistance of Mycobacterium tuberculosis to anti-tuberculosis drugs in the central region of Thailand. The International Journal of Tuberculosis and Lung Disease 1997;1(4): p. 299-301.

12. Shrestha B, Nehar A, Breyer U. The pattern of anti-tuberculosis drug resistance in patients treated at an urban $\mathrm{TB}$ clinic in Kathmandu Valley. J Nepal Med Assoc TB special 1996;117:36-40

13. Rijal KR, Ghimire P, Bam DS, Rijal B. An epidemiological study of anti-tuberculosis drug resistance pattern in the pulmonary tuberculosis patients visiting National Tuberculosis Centre. In: A Dissertation submitted to the Central Department of Microbiology, Tribhuvan University, Kirtipur, Kathmandu, Nepal, 2004.

14. Al-Marri MRHA. Pattern of mycobacterial resistance to four anti-tuberculosis drugs in pulmonary tuberculosis patients in the state of Qarter after the implementation of DOTS and limited expatriate screening Programme. The international journal of Tuberculosis and Lung Disease 2001;5(12):1116-1121.

15. Kuban C, Bercion R, Noeske J, Cunin P, Nkamsse P, Ngo Niobe S. Anti-tuberculosis drug resistance in the West Province of Cameroon. The International Journal of Tuberculosis and lung Disease 2002;4(4):356-60. 
16. Chhetri G, Sharma AP, Rijal BP. Prevalence of Tuberculosis among the suspected patients visiting Tribhuvan University Teaching Hospital and their Antimicrobial Resistance Pattern. In: A Dissertation submitted to the Central Department of Microbiology, Tribhuvan University, Kirtipur, Katmandu, Nepal, 2001 .

17. Bhattarai NR, Ghimire P, Shrestha B and Rijal BP. Anti-tuberculosis treatment resistant in pulmonary tuberculosis patients visiting German-Nepal Tuberculosis Project,Kalimati,Kathmandu. In: A Dissertation submitted to the Central Department of Microbiology, Tribhuvan University, Kirtipur, Kathmandu, Nepal, 2003.

18. Warndeoff DK, Yates M, Ngwira B, Chaguluka S, Jenkins PA, Drobniewski F. Trends in anti-tuberculosis drug resistance in Karonga District Malawi. The International journal of Tuberculosis and lung disease 2002;4(8):752-7
19. Smith I. Gender and Tuberculosis in Nepal. J Nepal Med Assoc TB special 1996:24(117):49-58.

20. Leung CC, Tam CM and Chan SL (2001) Efficacy of the BCG revaccination programme in a cohort given $\mathrm{BCG}$ vaccination at birth in Hong Kong. The International Journal of Tuberculosis and Lung Disease 2001;5(8):717-23.

21. National Tuberculosis Control Programme Annual Report, Thimi Bhaktapur: National Tuberculosis centre; 2002-2003

22. World Health Organization. TB: A clinical manual for South East Asia. Geneva; World Health Organization: 1997.

23. World Health Organization. Anti-tuberculosis drug resistance in the world: Prevalence and trends. Geneva; World Health Organization: 2000. 